\title{
S/TEM Characterization of Vertical Heterostructures Formed by Mono- to Multi- layer Graphene and WSe2
}

Saiphaneendra Bachu ${ }^{1}$, Benjamin Huet ${ }^{1}$, Danielle Reifsnyder Hickey ${ }^{2}$, Chenhao Qian ${ }^{1}$, Joan Redwing ${ }^{1}$ and Nasim Alem ${ }^{3}$

${ }^{1}$ Pennsylvania State University, United States, ${ }^{2}$ Pennsylvania State University, Pennsylvania, United States, ${ }^{3}$ Pennsylvania State University, Washington, District of Columbia, United States

Two-dimensional (2D) transition metal dichalcogenides (TMD) are emerging semiconductor materials in the field of nano optoelectronics owing to their desirable physical and electronic properties [1]. They are touted to replace silicon in the sub-5 $\mathrm{nm}$ regime where silicon suffers from quantum confinement effects [2]. Hence, there is a great interest in growing large area defect-free electronic grade TMD materials towards target optoelectronic applications. A recent development in the synthesis of TMD materials is the use of $2 \mathrm{D}$ growth substrates such as graphene and $\mathrm{hBN}$ as alternatives to traditional sapphire and silicon. Compared to the traditional substrates, 2D substrates offer potential advantages such as step edge free flat surfaces, absence of dangling bonds etc. [3]. Moreover, 2D substrates could enable true van der Waals epitaxy with the as-grown TMD with relaxed lattice matching conditions [4]. In addition, usage of 2D substrates could lead to the formation of $2 \mathrm{D}$ vertical heterostructures with enhanced optoelectronic properties and exciting physical phenomena [5, 6]. For example, H. Buch et al. have observed superlubricity in epitaxial graphene-WS2 vertical heterostructures [6].

In this work, we investigate the nucleation of $\mathrm{WSe}_{2}$ synthesized on mono- to multi-layer graphene via MOCVD process. The mono- to multi-layer graphene was first prepared using chemical vapor deposition (CVD) technique on $\mathrm{Cu}$ substrate and then transferred to sapphire substrate prior to the WSe2 growth. Asgrown heterostructures are characterized using scanning/transmission electron microscopy (S/TEM) imaging to understand the atomic structure of the heterostructures, epitaxy between graphene and WSe2 layers and the role of defects in graphene as the underlying mechanism behind the nucleation of $\mathrm{WSe}_{2}$ on Graphene.

Figure 1a shows a scanning electron microscope (SEM) image of WSe2 triangles ( $500 \mathrm{~nm})$ synthesized on CVD grown multilayer graphene after the MOCVD step. Layer numbers of graphene are labeled in the image. It can be observed that the nucleation density of $\mathrm{WSe}_{2}$ triangles is increasing with the graphene layer number. Selected area diffraction (SAD) pattern obtained from the as-grown heterostructures in monolayer graphene area is presented in Figures $1 \mathrm{~b}$. It is evident that WSe2 triangles (green hexagon) maintain an epitaxial relationship with the graphene layer (blue hexagon). Figures 2 shows the atomic resolution images of graphene and WSe2. While Figure 2a is a monochromated high-resolution TEM image taken from monolayer graphene region, Figure $2 b$ is an atomic resolution high-angle annual darkfield (HAADF)-STEM image taken from a WSe2 triangle. The presentation will further uncover the role of the substrate and epitaxy, graphene layer number, and substrate defects on the nucleation density of $\mathrm{WSe}_{2}[7]$. 

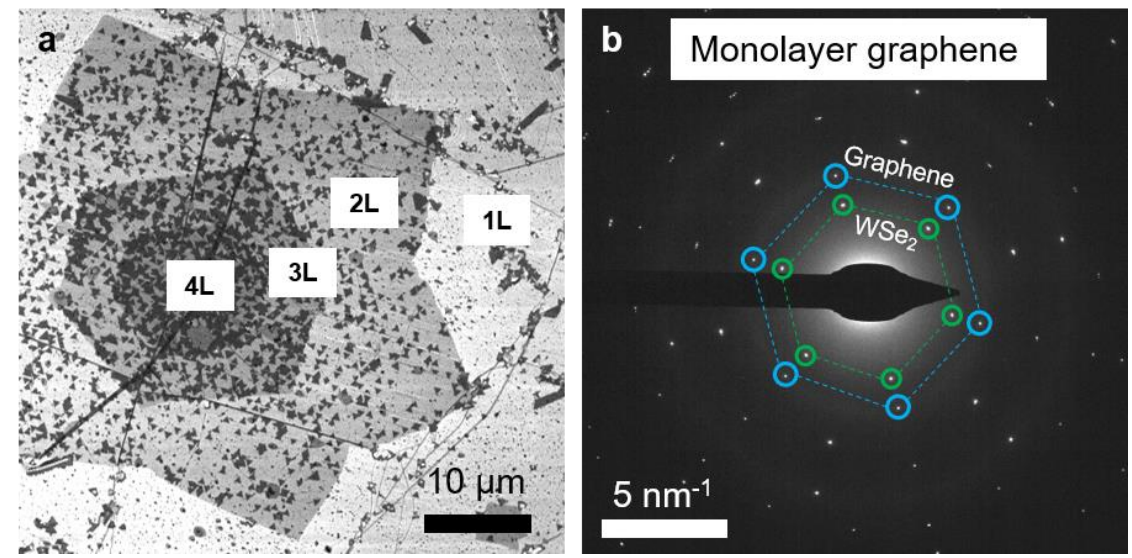

Figure 1. (a) SEM image of $\mathrm{WSe}_{2}$ triangles grown on multilayer CVD graphene; the labels indicate the number of graphene layers and (b) SAD pattern obtained from heterostructures grown on the monolayer graphene area showing epitaxy between graphene and $\mathrm{WSe}_{2}$.
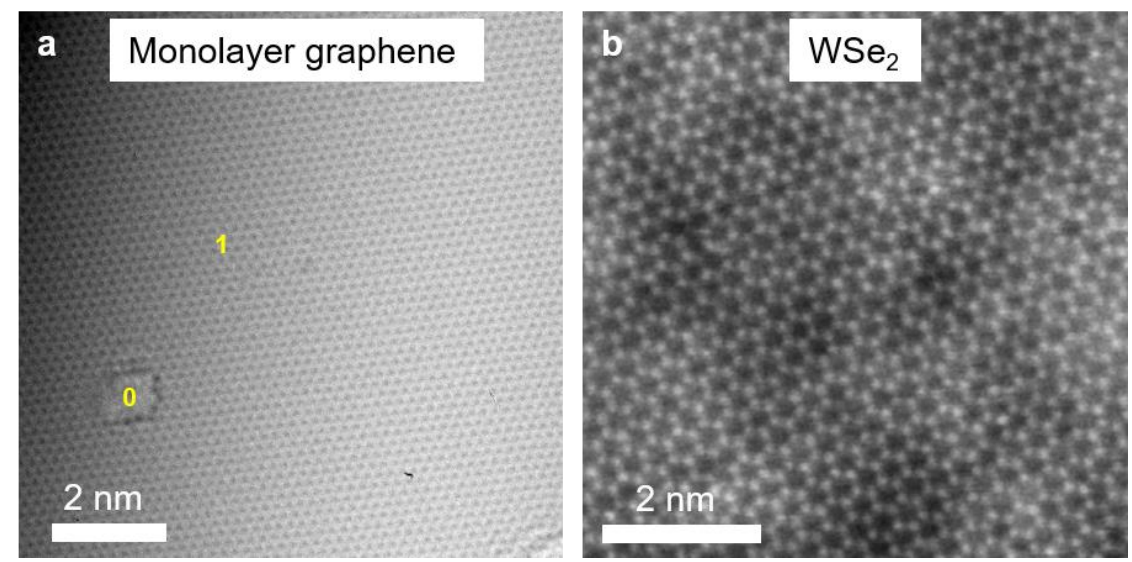

Figure 2. (a) Monochromated HRTEM image from monolayer graphene region; the numbers in the image indicate the number of graphene layers and (b) atomic resolution HAADF-STEM image of WSe 2 grown on the monolayer graphene region.

\section{References}

[1] Jariwala, Deep, et al., ACS nano 8.2 (2014): 1102-1120.

[2] Tsutsui, Gen, et al., IEEE Transactions on nanotechnology 4.3 (2005): 369-373.

[3] Zhang, Xiaotian, et al., ACS nano 13.3 (2019): 3341-3352.

[4] Bianco, G. V., et al., RSC advances 5.119 (2015): 98700-98708.

[5] Lin, Yu-Chuan, et al., Nano letters 14.12 (2014): 6936-6941.

[6] Büch, Holger, et al., Nano Research 11.11 (2018): 5946-5956.

[7] This work was supported by the National Science Foundation (NSF), in part under the CAREER program (DMR-1654107), in part by the program EFRI 2-DARE: 2D Crystals by Activated Atomic Layer Deposition (EFRI-1433378), and in part by the Penn State 2D Crystal Consortium-Materials Innovation Platform (2DCC-MIP) under NSF cooperative agreement DMR-1539916. 